\title{
Erratum to: Neuroprotective role of taurine during aging
}

\author{
Abdeslem El Idrissi • Chang Hui Shen • \\ William J. L'Amoreaux
}

Published online: 20 December 2013

(C) Springer-Verlag Wien 2013

\section{Erratum to: Amino Acids (2013) 45:735-750 DOI 10.1007/s00726-013-1544-7}

This article is part of the Special Issue "Clinical significance of taurine" edited by S. Schaffer while it was published already in print in Volume 45, Issue 4.

\begin{abstract}
Aging of the brain is characterized by several neurochemical modifications involving structural proteins, neurotransmitters, neuropeptides and related receptors. Alterations of neurochemical indices of synaptic function are indicators of age-related impairment of central functions, such as locomotion, memory and sensory performances. Several studies demonstrate that ionotropic GABA
\end{abstract}

The online version of the original article can be found under doi:10.1007/s00726-013-1544-7.

A. El Idrissi $(\bowtie) \cdot C$. H. Shen · W. J. L'Amoreaux

Department of Biology, Center for Developmental

Neuroscience, City University of New York Graduate School, Staten Island, NY 10314, USA

e-mail: abdeslem.elidrissi@csi.cuny.edu

A. El Idrissi - W. J. L'Amoreaux

The Graduate Center, Program in Biology-Neurosciences,

The City University of New York, 365 Fifth Avenue, New York, NY 10016, USA

\section{H. Shen}

The Graduate Center, Program in Biology-Molecular, Cellular and Developmental Biology, The City University of New York, 365 Fifth Avenue, New York, NY 10016, USA

W. J. L'Amoreaux

The Graduate Center, Program in Biochemistry, The City

University of New York, 365 Fifth Avenue, New York,

NY 10016, USA receptors, glutamate decarboxylase (GAD), and somatostatinergic subpopulations of GABAergic neurons are markedly decreased in experimental animal brains during aging. Additionally, levels of several neuropeptides coexpressed with GAD decrease during aging. Thus, the agerelated decline in cognitive functions could be attributable, at least in part, to decrements in GABA inhibitory neurotransmission. In this study, we showed that chronic supplementation of taurine to aged mice significantly ameliorated the age-dependent decline in spatial memory acquisition and retention. We also demonstrated that concomitant with the amelioration in cognitive function, taurine caused significant alterations in the GABAergic and somatostatinergic system. These changes included (1) increased levels of the neurotransmitters GABA and glutamate, (2) increased expression of both isoforms of GAD (65 and 67) and the neuropeptide somatostatin, (3) decreased hippocampal expression of the $\beta 3$ subunits of the $\mathrm{GABA}_{\mathrm{A}}$ receptor, (4) increased expression in the number of somatostatin-positive neurons, (5) increased amplitude and duration of population spikes recorded from CA1 in response to Schaefer collateral stimulation and (6) enhanced paired pulse facilitation in the hippocampus. These specific alterations of the inhibitory system caused by taurine treatment oppose those naturally occurring in the aging brain, suggesting a protective role of taurine in this process. An increased understanding of age-related neurochemical changes in the GABAergic system will be important in elucidating the underpinnings of the functional changes of aging. Taurine supplementation might help forestall the age-related decline in cognitive functions through interaction with the GABAergic system.

Keywords Taurine - Aging - Somatostatin - GABA · GAD - Passive avoidance 